\title{
Médiévales
}

Langues, Textes, Histoire

66 | printemps 2014

Harmonie Disharmonie

\section{L'harmonie des sphères et la danse dans le contexte clérical au Moyen Âge}

Dance and the Harmony of the Spheres in the Medieval Clerical Context

\section{Philip Knäble}

\section{(2) OpenEdition}

1 Journals

Édition électronique

URL : https://journals.openedition.org/medievales/7202

DOI : $10.4000 /$ medievales.7202

ISSN : $1777-5892$

Éditeur

Presses universitaires de Vincennes

Édition imprimée

Date de publication : 30 juin 2014

Pagination : 65-80

ISBN : 978-2-84292-405-8

ISSN : 0751-2708

\section{Référence électronique}

Philip Knäble, "L'harmonie des sphères et la danse dans le contexte clérical au Moyen Âge », Médiévales [En ligne], 66 | printemps 2014, mis en ligne le 05 juillet 2016, consulté le 22 avril 2022. URL : http://journals.openedition.org/medievales/7202 ; DOI : https://doi.org/10.4000/medievales. 7202 


\section{Philip Knäble}

\section{L'harmonie des sphères et la danse dans le contexte clérical au Moyen Âge}

C'est avec plaisir, Monsieur, que je vous envoye le petit détail que vous m'avez demandé, je m'attends bien que vous en ferez part aux Auteurs du Mercure de France, qui pourront réjoüir encore une fois le Public aux dépens de la simplicité \& de l'ignorance de nos Ancêtres ${ }^{1}$.

L'ignorance des ancêtres est un sujet fréquent dans plusieurs articles du Mercure de France au début du XVIII ${ }^{\mathrm{e}}$ siècle. Quelques auteurs décrivent en détail des fêtes et des cérémonies du Moyen Âge tardif, qui leur semblent extravagantes et sans raison. Parmi eux on compte l'Abbé Jean Lebeuf (1687-1760), chanoine d'Auxerre, un homme savant et enthousiaste de la réforme liturgique ${ }^{2}$. Lebeuf s'intéresse beaucoup aux rituels de cette époque, qu'il considère comme des curiosités. Il relate en particulier certaines pratiques étonnantes dans les églises de France. Il décrit la Fête de l'Âne, une «coutume grossiere $\&$ gothique $^{3}$ » et les abus ecclésiastiques à la Fête des Fous et à la Fête de l'évêque des Innocents. Ce sont surtout les fêtes, les jeux et les danses qui attirent l'attention de Lebeuf. En mai 1726, il raconte dans un article du Mercure de France une autre «grande bizarrerie ${ }^{4} »$ : il s'agit d'une ancienne danse ecclésiastique, qui se pratiquait dans la nef de la cathédrale d'Auxerre le jour de Pâques jusqu'à son abolition par

1. «Lettre écrite d'Évreux le 6 Janvier 1726 par M. L. à M. A. sur une ancienne \& singuliere Ceremonie de cette ville», Le Mercure de France, 10 (1726), p. 694. Je remercie Colin Arnaud pour avoir corrigé mes faiblesses linguistiques.

2. Sur Lebeuf, $c f$. X. Bisaro, L'Abbé Lebeuf, prêtre de l'histoire, Turnhout, 2011, p. 7-37.

3. «Lettre écrite à M... sur l'explication d'un terme de la basse Latinité», Le Mercure de France, 9 (1725), p. 1601.

4. J. Lebeuf, «Explication d'un terme de la basse Latinité », Le Mercure de France, 10 (1726), p. 911-925. 
le parlement de Paris en $1538^{5}$. À la fin de son article, Lebeuf cite une description de cette cérémonie tirée d'un manuscrit du $\mathrm{XVI}^{\mathrm{e}}$ siècle $^{6}$.

Après avoir reçu la pelote [une sorte de ballon], le doyen ou quelqu'un pour lui, portant sur sa tête une almutia propre à la Fête de Pâques, coiffure que les autres portaient également, entonnait l'antienne des Victimae Paschali laudes. Puis le doyen, en prenant le ballon de sa main gauche, dansait un tripudium accompagné par les sons rythmiques de l'antienne, alors que les autres, se tenant par la main, dansaient une chorea autour du dédale. En même temps le Doyen donnait ou lançait le ballon aux autres qui dansaient comme des serpents à coté de lui. Ainsi était le jeu, et l'orgue jouait le rythme de la danse.

Auxerre n'était pas le seul lieu où les chanoines «dansaient ${ }^{7}$ » dans l'église pendant une fête chrétienne importante, même si la combinaison d'une danse et d'un lancer de ballon sur le labyrinthe de la nef semble un cas unique. Plusieurs registres des chapitres cathédraux en France mentionnent des danses ou des jeux de pelote ${ }^{8}$. Claude-François Ménestrier, un jésuite

5. Sur la danse à Auxerre, $c f$. C. Wright, The Maze and the Warrior. Symbols in Architecture, Theology and Music, Cambridge, 2001, p. 139-145; U. Zellmann, «Lusus erat. Tanz und Spiel auf dem Kirchenlabyrinth in der Kathedrale von Auxerre », dans H. BritTNACHER éd., Labyrinth und Spiel. Umdeutungen eines Mythos, Göttingen, 2007, p. 3674; M. EISENBERG, «Performing the Passion. Music, Ritual, and the Eastertide Labyrinth», Revista Transcultural de Música/Transcultural Music Review, 13 (2009), sans pagination; P. KNÄBLE, «Ausgetanzt - Schwindende Akzeptanz eines kirchlichen Initiationsrituals im Spätmittelalter», dans A. BütTneR, A. Sсhmidt, P. Töвelmann éd., Grenzen des Rituals. Wirkreichweiten - Geltungsbereiche - Forschungsperspektiven, Cologne, 2014, p. 27-48.

6. «Acceptâ pilotâ à proselyto seu tirone Canonico, Decanus, aut alter pro eo olim gestans in capite amutiam a pretique pariter, aptam diei Festo Pascha Prosm antiphonat gestans in capite al pilonabat quae incipit Victinae Paschal laces: tum laeva pilotam apprehendens, ad Prosae decantatae numerosos sonos tripudium agebat, ceteris manu prehensis choream circa daedalum ducentibus, dum interim per alternas vices pilota singulis aut pluribus ex choribaudis à Decano serti in speciem tradebatur aut jaciebatur. Lusus erat \& organi ad choreae numeros » (J. Lebeuf, «Explication d'un terme...», p. $921 s q$.).

7. Il est très difficile de traduire le vocabulaire latin médiéval de la danse, comme chorus/chorea, tripudium et saltatio. Celui-ci est souvent peu clair, notamment pour des termes comme chorus et tripudium, dont on ne sait pas toujours s'ils décrivent des danses ou 'ils désignent des chants ou une expression de joie. Il y a des exemples où l'on devine par co con le conte che . pour les mouvements des chanoines, tripudium pour les mouvements du doyen et saltatio pour la danse entière. Pour cette raison je traduis ces termes par le mot français «danse». $C f$. D. LA RuE, «Tripudium. Its Use in Sources from 200 BCE to 1600 CE », The Religion and Arts Program, 7 (1995), p. 25-29; H. SPANKE, «Tanzmusik in der Kirche des Mittelalters», Neuphilologische Mitteilungen, 31 (1930), p. 151

8. Sans doute à Sens, Chartres, Besançon, Nîmes, Nevers, Narbonne; probablement aussi à Amiens, Rouen, Moissac, Viennes, Salins: $c f$ G. RoHmann, Tanzwut. Kosmos, Kirche qui écrit une histoire du ballet à la fin du XVII ${ }^{\mathrm{e}}$ siècle, raconte qu'il connaissait encore des danses dans les églises ${ }^{9}$ :

J'ay vû encore en quelques Eglises le jour de Pâques les Chanoines prendre par la main les Enfants de Choeur, \& en chantant des Hymnes de rejouissance danser dans l'Eglise: pour ne rien dire des coûtumes scandaleuses, que la simplicité avoit introduites il y a deux ou trois siècles...

Ménestrier décrit une pratique, qui est déjà en train de disparaitre au $\mathrm{XVII}^{\mathrm{e}}$ siècle. Cinquante ans plus tard, Lebeuf et ses contemporains ne connaissent plus ces danses qui ne leur semblent que des bizarreries. À la fin du XVIII ${ }^{\text {e }}$ siècle, le souvenir des danses dans les églises s'est totalement estompé. Le romantisme du XIX ${ }^{\mathrm{e}}$ siècle, qui fait amplement référence au Moyen Âge, ne s'intéresse pas à la danse des clercs, et l'Église catholique, en conflit avec l'État en France et en Allemagne, n'a aucun motif de redécouvrir cet aspect peu glorieux de son histoire. À cette époque se développe l'image d'une Église médiévale complètement opposée à la danse, image qui influence le jugement de la recherche jusqu'à nos jours ${ }^{10}$. D'une part, on trouve des sermons et des traités de clercs contre la danse et l'on peut fréquemment attester l'image d'une danse disharmonique au Moyen Âge; mais d'autre part, à la même époque, il existe des clercs qui estiment la danse moins dangereuse, voire même bénéfique ${ }^{11}$. La danse est donc sujette à un discours ambivalent. Inspirée des exemples bibliques de David ou de Myriam et faite pour honorer Dieu, la danse est un moyen de symboliser, ou même d'atteindre, l'harmonie céleste. Motivée par la volupté et des suggestions diaboliques, les mouvements disharmoniques des danseuses et danseurs courent le risque de déformer l'ordre social et donc l'harmonie divine ${ }^{12}$. Je veux montrer que, sous certaines conditions, des danses étaient bel et bien pratiquées par le clergé dans les églises du Moyen

und Mensch in der Bedeutungsgeschichte eines mittelalterlichen Krankheitskonzeptes, Göttingen, 2013, p. $243 s q$.

9. C.-F. Menestrier, Des ballets anciens et modernes selon les règles du théâtre, Paris, 1682 , p. II.

10. Cf. A. Angenendt, Geschichte der Religiosität im Mittelalter, Darmstadt, 2009, p. 421 : «Zur Musik gehört der Tanz. [.. ] Aber auch hiergegen richteten die Christen schärfste pritik. Ihnen wurde der Tanz "schlechthin zum Ausdruck heidnischer Frömmigkeit". Das Kritik. Ihnen wurde der Tanz "sch
Mittelalter sah darin Teufelswerk».

11. Cf. A. Arcangeli, «Dance under Trial: The Moral Debate», Dance research, 12 (1994), p. 127-155; M. Ruel, Les Chrétiens et la danse dans la France moderne. XVIXVIII' siècle, Paris, 2006, p. 71-122

12. $C f$. J. Zimmermann, Teufelsreigen-Engelstänze. Kontinuität und Wandel in mittelalterlichen Tanzdarstellungen, Francfort, 2007, p. 49-148, 289-336; G. RонмамN, Tanzwut..., p. 212-239. 
Âge et que, loin d'être des pratiques ridicules, elles s'inspiraient d'une réception chrétienne du concept platonicien de l'harmonie des sphères.

\section{L'harmonie des sphères}

La philosophie de Platon contient de nombreuses allusions à la danse. Graham Pont la qualifie pour cette raison de «philosophy of dance ${ }^{13}$ », en montrant les rapports complexes de Platon avec la musique et la danse. Pour l'éducation des jeunes Grecs la pratique de la danse est tellement importante que Platon qualifie les gens sans éducation de achoreutos - ceux qui ne savent pas danser ${ }^{14}$. Les idées de Platon concernant la danse, qu'il explique dans le dernier chapitre de La République et surtout dans le Timée, sont toujours en relation avec l'harmonie de sphères. Platon décrit les mouvements des astres comme une danse divine, une danse cosmique chorégraphiée harmonieusement.

Cette image de la danse est fortement influencée par l'École pythagoricienne qui essaye de découvrir l'harmonie des sphères par des proportions mathématiques (en combinant les chiffres un, deux, trois et quatre). Cette combinaison est la base de la théorie musicale pythagoricienne, dans laquelle la musique harmonieuse des astres en mouvement trouve sa correspondance dans une musique ou une danse mesurées ${ }^{15}$.

La philosophie de Platon reste importante durant toute l'Antiquité, mais c'est surtout au $\mathrm{III}^{\mathrm{e}}$ siècle après Jésus-Christ qu'elle acquiert une ampleur particulière grâce aux philosophes néoplatoniciens ${ }^{16}$. À cette époque la philosophie néoplatonicienne a non seulement une grande influence dans le culte impérial, mais aussi dans la théologie chrétienne. Bien qu'il y ait plusieurs manifestations de cette philosophie, Gregor Rohmann la divise d'une façon générale en deux écoles : d'une part celle de Plotin, qui présente plutôt une interprétation allégorique ou spirituelle de la danse, d'autre part celle de Jamblique, qui préfère une sorte de communication rituelle par la pratique ${ }^{17}$. L'Église est l'héritière de ces deux tendances qui débattent pendant toute l'Antiquité tardive pour savoir si la danse est juste l'image

13. G. Pont, «Plato's Philosophy of Dance», dans J. Nevile éd., Dance, Spectacle, and the Body Politick, 1250-1750, Bloomington, 2008, p. 267.

14. Cf. ibid. p. 267-271.

15. Cf. G. Berghaus, «Neoplatonic and Pythagorean Notions of World Harmony and Unity and their Influence on Renaissance Dance Theory », Dance Research, 10 (1992), p. 4448 .

16. Pour la réception de l'image de la danse chez Platon, $c f$. J. Mitler, Measures of Wisdom. The Cosmic Dance in Classical and Christian Antiquity, Toronto, 1986.

17. Cf. G. Rohmann, Tanzwut..., p. 118 d'une méditation et d'un souci de soi ou si les fidèles peuvent communiquer avec Dieu par la pratique corporelle de la danse.

À coté de la discussion sur l'esprit et le corps, les auteurs chrétiens différencient aussi la motivation des danses. Le commentaire d'Ambroise de Milan sur l'évangile de Luc montre bien l'argumentation ${ }^{18}$ :

David a chanté pour que nous suspendions nos harpes aux saules. Il a chanté et il a dansé devant l'arche du Seigneur, non pour folâtrer mais par religion. Donc ce qui est indiqué, ce ne sont pas les bonds d'un corps infléchi en contorsions de saltimbanques, mais l'agilité d'un esprit éveillé, d'un corps consacré.

La danse est donc une activité légitime si elle est motivée par la foi. Cette danse consiste en des mouvements harmonieux, ce qui implique certes des gesticulations, mais des gesticulations mesurées. Les mouvements des danses illégitimes sont au contraire contorsionnés, non réglés, comme ceux des histrions au cirque ${ }^{19}$. Les Pères de l'Église les comparent souvent avec les danses des bacchantes, qui sont considérées comme des mouvements disharmonieux. La danse sacrée, en revanche, est harmonieuse parce qu'elle reflète l'harmonie divine.

Clément d'Alexandrie est l'un des premiers auteurs chrétiens qui reprend l'idée de l'harmonie des sphères et qui discute de l'intégration de la danse à la prière et aux pratiques de dévotions au début du $\mathrm{III}^{\mathrm{e}}$ siècle $^{20}$. Un siècle plus tard, on trouve beaucoup plus fréquemment la danse comme partie intégrante de la dévotion. C'est une homélie qui propose: «Mais qu'offrirons-nous au martyr qui soit digne de lui? [...] Si vous voulez, célébrons en son honneur nos danses accoutumées ${ }^{21}$.» À la même époque, Basile de Césarée, un des Pères de l'Église du $\mathrm{IV}^{\mathrm{e}}$ siècle, écrit sur la danse: «Que pourrait être de plus bénit que d'imiter sur terre les danses des anges $^{22}$ ?» Ce sont deux exemples qui montrent que les théologiens de cette époque considèrent la danse comme une forme légitime de dévotion.

18. «Cantavit David, ut in salicibus nostra suspenderemus organa, cantavit ipse et ante arcam domini non pro lascivia, sed pro religione saltavit. Ergo non histrionicis motibus sinuati corporis saltus, sed inpigra DE MILAN, Traité sur l'évangile de S. Luc, Paris, 1971, t. I, p. 229 sq.). 19. Cf. C. AnDRESEN, «Altchristliche Kritik am Tanz - ein Ausschnitt aus dem Kampf der Alten Kirche gegen heidnische Sitte», dans H. FrohNes éd., Kirchengeschichte als Missionsgeschichte, t. 1., Die Alte Kirche, Munich, 1974, p. 344-376.

20. Cf. J.Zimmermann, Teufelsreigen..., p. 100.

21. Cité par M. SAHLIN, Étude sur la carole médiévale. L'origine du mot et ses rapports avec l'Église, Uppsala, 1940, p. 138.

22. "Quid itaque beatius esse poterit quam in terra tripudium angelorum imitari? (Ibid.) 
Les théologiens transposent donc l'idée de l'harmonie des sphères au contexte chrétien. Les mouvements des astres deviennent les danses des martyrs ou des anges qui entourent Dieu et le célèbrent par leurs danses. C'est surtout à travers les œuvres d'Augustin, de Macrobe, de Boèce et du Pseudo-Denys l'Aréopagite que la philosophie néoplatonicienne et l'image des anges dansants sont reprises au Moyen Âge. Le Pseudo-Denys divise les armées célestes en neuf hiérarchies qui entourent Dieu, le centre de la connaissance, par des rondes. Il explique qu'il existe, pour les clercs, la possibilité de partager la connaissance des anges s'ils parviennent, par la contemplation, à participer aux danses des anges ${ }^{23}$. Les œuvres de ces auteurs sont des lectures essentielles pour l'éducation dans les écoles cathédrales, dans lesquelles elles propagent l'idée de l'harmonie des sphères.

\section{La danse dans les écoles cathédrales}

Avant le succès des universités et la redécouverte de la philosophie d'Aristote au XIII ${ }^{\text {e }}$ siècle, les écoles monastiques et cathédrales sont les centres du savoir et de la recherche. Dans les écoles cathédrales, la philosophie de Platon - et à travers celle-ci l'idée de l'harmonie des sphères - connaît une renaissance. À coté des œuvres d'Augustin, de Boèce, de Macrobe et du Pseudo-Denys l'Aréopagite, les clercs lisent le Timée de Platon dans la traduction latine de Cicéron et, principalement, dans celle de Calcidius accompagnée de son commentaire, toutes deux partielles. Le $\mathrm{XII}^{\mathrm{e}}$ siècle représente l'apogée de la réception de Platon, illustré entre autres par le nombre de copies du Timée ${ }^{24}$. Pour les théologiens de cette époque, notamment pour les maîtres de l'école de Chartres, mais aussi pour Pierre Abélard (1142) ou Richard de Saint-Victor (1173), la philosophie platonicienne est une référence majeure de leurs réflexions théologiques ${ }^{25}$.

Dans son œuvre Mysticae Adnotationes in Psalmos, Richard de Saint-Victor reprend l'idée de l'imitation de la danse des anges et invite à la danse spirituelle ${ }^{26}$ :

23. Cf. G. Rohmann, Tanzwut..., p. 121-126.

24. Cf. R. Klibansky, The Continuity of the Platonic Tradition during the Middle Ages, Londres, 1950, p. 28 ; T. RICKLIN, «Plato im zwölften Jahrhundert. Einige Hinweise zu seinem Verschwinden», dans S. Gersh, M. Hoenen éd., The Platonic Tradition in the Middle Ages. A Doxographic Approach, Berlin, 2002, p. 139-141 et 161-163.

25. F. BezNer, «Simmistis veri. Das Bild Platons in der Theologie des zwölften Jahrhunderts », dans S. Gersh, M. HoEnen éd., The Platonic Tradition in the Middle Ages..., p. $93-95$ et $103-107$

26. «Spirituales itaque, non corporales saltus in hac prophetica sententia quaeramus, et quales denique decebat vel Spiritum sanctum docere, vel prophetam describere. Corporalis saltus, est totum corpus a terra suspendere. Spiritualis saltus, spiritum et totum quod spiritus est a terrenis alienare. Corporalis saltus, est terrae tactum usquequaque deserere, et totius
C'est pourquoi il convenait que nous cherchions des danses spirituelles et non des danses corporelles dans cette sentence prophétique, et enfin que celles-ci enseignent le Saint-Esprit ou bien qu'elles décrivent le prophète. La danse corporelle, c'est suspendre tout le corps de la terre. La danse spirituelle, c'est aliéner de la terre l'esprit et tout ce qui est spirituel. La danse corporelle consiste à perdre en tous points le contact avec la terre, et à libérer dans le vide les membres de tout le corps. La danse spirituelle consiste à dépasser la raison, et, après avoir quitté les plus bas soubassements, à traverser le tout dans la contemplation des invisibles.

Richard établit la différence entre la danse spirituelle et la danse corporelle. Alors que la danse corporelle nécessite de se lever de la terre par un saut pour y revenir, la danse spirituelle signifie lâcher tout lien temporel par une contemplation invisible et immobile ${ }^{27}$. C'est seulement la danse spirituelle qui mène à l'union mystique avec Dieu, parce que le danseur parvient à s'élever de la terre grâce à elle. Les danses corporelles, au contraire, restent toujours, malgré leurs sauts, reliées à la terre, c'est-à-dire à un monde vain et vide. Ces danses n'arrivent pas à créer un mouvement harmonieux, elles sont au contraire proches des danses disharmonieuses, contre lesquelles plusieurs théologiens médiévaux alertent les fidèles, comme le prédicateur franciscain Jacques de Vitry (1240): «La danse est un cercle dont le centre est le Diable ${ }^{28}$.» La réception de la conception platonicienne de la danse se retrouve encore au XIII ${ }^{\mathrm{e}}$ siècle dans le Roman de la Rose, commencé vers 1220 par Guillaume de Lorris et continué par Jean de Meun vers 1270 1280. À côté des nombreux sujets, le roman traite de questions théologiques et philosophiques discutées dans les écoles cathédrales du Bassin parisien et à l'Université de Paris, où Jean de Meun reçoit sa formation intellectuelle ${ }^{29}$. James Miller a bien montré que le sujet de la danse traverse le roman dès le début - avec la carole du jardin de Déduit - jusqu'à sa fin. De plus, Guillaume de Lorris commence le roman en mentionnant le commentaire de Macrobe sur le Songe de Scipion de Cicéron ${ }^{30}$. Il amorce le lien entre la danse et la philosophie néoplatonicienne et Jean de Meun reprend ce sujet

corporis membra per inane librare. Spiritualis saltus est mente excedere, et infimis in imo relictis in invisibilium contemplationem totum transire» (Ricardus SANCTI Victori, Mysticae Adnotationes in Psalmos, psaume CXIII, PL 196, col. 338).

27. Cf. J. Zimmermann, Teufelsreigen..., p. $129 \mathrm{sq}$.

28. «Chorea enim circulus est, cuius centrum est diabolus» (JACQUES DE VITRY, Sermone vulgares domini Jacobi Vitricensis, Paris, BnF, lat. 17509, $\left.\mathrm{f}^{\circ} 146 \mathrm{r}^{\circ}\right)$.

29. Cf. I. Wer, Intellectual Culture in Medieval Paris. Theologians and the University c. 1100-1330, Cambridge, 2012, p. 357-373.

30. Guillaume de Lorris et Jean de Meun, Le Roman de la Rose, v. 7-10: «Un auctor qui ot non Macrobes; Qui ne tient pas songes à lobes; Ançois escrist l'avision, Qui avint au roi Scipion» (éd. K.-A. Отт, Munich, t. I, 1976, p. 78). 
de plusieurs façons en commentant Calcidius, Martianus Capella et Boèce ${ }^{31}$. Jean de Meun reprend aussi la scène de la danse dans le jardin de Déduit à la fin du roman. Contrairement à son prédécesseur, il considère la danse comme un mouvement vain et corporel qui mène directement à l'enfer et parle des «queroles qui faillirent ${ }^{32} »$. Uniquement ceux qui ont la chance de participer à la danse pure et harmonique, pratiquée par les anges, peuvent échapper à la damnation ${ }^{33}$. Jean de Meun fait allusion à la différence entre la beata chorea et la chorea mundi qui était déjà la référence chez Richard de Saint-Victor.

C'est surtout le personnage de Genius qui, en discutant des diverses interprétations de la danse, réfléchit sur la relation entre la danse et son rôle dans l'harmonie céleste. Genius considère toutes les sortes de danses comme faisant partie d'un plan divin et, selon Miller, il élargit le concept du Pseudo-Denys l'Aréopagite selon lequel les danses terrestres peuvent être la contrepartie des rondes angéliques. Jean de Meun s'inspire, pour le personnage de Genius, des écrits des maîtres de l'école cathédrale de Chartres, notamment du De planctu naturae d'Alain de Lille et du De mundi universitate sive megacosmus et microcosmus de Bernard Silvestre ${ }^{34}$. L'école de Chartres était un centre de la réception de Platon: au XII ${ }^{\mathrm{e}}$ siècle, les discussions et les enseignements de ses maîtres portaient sur des thèmes comme la cosmologie ou la création du monde. À Chartres, le quadrivium était le fondement des études; l'astronomie et la musique aidaient en particulier les élèves à comprendre la philosophie.

L'importance de la musique est visible dans l'architecture même de la cathédrale de Chartres. Le portail royal de la façade occidentale - l'entrée principale de la cathédrale - offre en effet une représentation des sept arts libéraux ${ }^{35}$. Sept femmes représentent les arts, chacune d'elles est accompagnée d'un philosophe antique : Aristote pour la dialectique, Cicéron pour la rhétorique, Euclide pour la géométrie, Boèce pour l'arithmétique, Ptolémée pour l'astronomie, Donat pour la grammaire et Pythagore pour la musique. D'après Martianus Capella et le Commentaire de Macrobe sur le Songe de Scipion de Cicéron, les sept femmes signifient aussi les muses associées aux sept planètes. Le mouvement harmonieux des planètes est repris par les voix harmonieuses des muses qui forment dans leur ensemble la ronde céleste. Le portail illustre donc une allégorie du concert céleste et

31. Cf. J. Miller, Choreia. Visions of the Cosmic Dance in Western Literature from Plato to Jean de Meun, Toronto, 1979, p. 518-528.

32. Guillaume de Lorris et Jean de Meun, Le Roman de la Rose, v. 20355 (t. III, 1979, p. 1084).

33. Cf. J. Miller, Choreia..., p. 532-535.

34. Ibid., p. 543-552.

35. Je suis ici l'interprétation de C. Hummel, Pythagoras und die Meister von Chartres, Darmstadt, 2007, p. 26-56. de l'harmonie des sphères ${ }^{36}$. Alors que six arts se trouvent sur l'archivolte extérieure, c'est seulement Pythagore qui se trouve sur l'archivolte intérieure, fournissant la base du demi-cercle d'anges et recevant donc une position particulière. Le personnage féminin joue du carillon et tient une cithare et un monocorde, trois instruments qui permettent une modulation exacte et mathématique de la musique. De plus, le monocorde est l'instrument que Pythagore présente comme outil de la transmission de la musique des sphères aux hommes. Boèce décrit également les rapports intimes entre la musique des astres et les cordes du monocorde ${ }^{37}$.

Le portail royal de Chartres a été construit au milieu du XII ${ }^{\mathrm{e}}$ siècle, alors que les différents maîtres de Chartres produisaient leurs nombreux écrits sur l'harmonie des sphères. La conception du portail est certainement influencée par les considérations théologiques et philosophiques de ces maîtres, surtout par le chancelier de l'école cathédrale, Thierry de Chartres $(1155)^{38}$. Thierry manifeste en effet un grand intérêt pour l'influence de Pythagore sur Platon. Il discute particulièrement de la combinaison entre les mathématiques et la musique pour trouver un moyen de découvrir l'harmonie. Un auteur anonyme, qui dédie un livre à Thierry, écrit: «À toi, Thierry, mon maître appliqué, par lequel l'âme de Platon renaît sans doute $^{39}$.»

Le poète et théologien Alain de Lille s'intéresse également à l'idée de l'harmonie des sphères dans son Anticlaudianus. Il y décrit les sept arts libéraux construisant un chariot pour voyager à travers les sphères vers Dieu, et montre comment la musique des sphères crée un lien entre microcosme et macrocosme ${ }^{40}$. L'influence de la philosophie néoplatonicienne est forte chez d'autres maîtres de l'école cathédrale. Bernard Silvestre, Guillaume de Conches et Bernard de Chartres écrivent tous un commentaire du Timée de Platon, et illustrent le rapport entre la musique, la danse et l'harmonie des sphères ${ }^{41}$. L'image de la danse harmonieuse des astres est donc fréquente à l'école de Chartres et probablement dans d'autres écoles cathédrales ${ }^{42}$. Il existe par conséquent une idée positive de la danse, mais il s'agit surtout

36. $C f$. M.-Th. D’AlvernY, «Les Muses et les sphères célestes», dans C. Henderson éd., Classical Mediaeval and Renaissance Studies in Honor of Berthold Louis Ullman, Rome, 1964, t. II, p. 7-9.

37. Cf. R. BragaRD, «L'harmonie des sphères selon Boèce», Speculum, 4 (1929), p. 208

38. Cf. T. Evers, Logos und Sophia. Das Königsportal und die Schule von Chartres, Kiel, 2011, p. 80-86, 97-101.

39. «Dir, Thierry, mein emsiger Meister, in dem ohne Zweifel Platons Seele wiedergeboren wurde» (C. HummeL, Pythagoras und..., p. 43).

40. Ibid., p. 36.

41. Cf. P. Dronke, The Spell of Calcidius. Platonic Concepts and Images in the Medieval West, Florence, 2008, p. 117-162.

42. Pour les cas de Sens et d'Auxerre, il manque des études précises. La réception des textes néoplatoniciens à l'école monastique d'Auxerre est du moins fréquente au $\mathrm{X}^{\mathrm{e}} \mathrm{s}$. 
d'une danse spirituelle et mystique, «c'est-à-dire » une danse non corporelle. Le lien entre cette image positive et la pratique des danses ecclésiastiques reste encore vague. Pour clarifier ce point, il faut prendre en compte les écrits des liturgistes de la même époque.

\section{Les liturgistes et la danse}

En même temps que la discussion dans les écoles cathédrales, plusieurs liturgistes mentionnent dans leurs écrits les pratiques de la danse et du jeu de ballon dans les grandes églises en France. Les clercs français Jean Beleth (après 1165) et Durand de Mende (1296) racontent que des évêques, et même des archevêques, pratiquent des jeux de ballon avec les chanoines de l'église cathédrale le jour de Pâques. L'évêque italien Sicard de Crémone (1215) précise qu'il faut considérer ces jeux de ballon comme des danses. Une copie de la Summa de officiis ecclesiasticis de Guillaume d'Auxerre (1231) ne mentionne plus le jeu, mais parle uniquement des danses de Pâques. Tous les auteurs se réfèrent aux écrits liturgiques d'Honorius Augustodunensis ${ }^{43}$, qui développe en même temps que l'école de Chartres une interprétation chrétienne de la danse des astres ${ }^{44}$.

Jean Beleth commence sa formation à l'abbaye de Tiron, à quarante kilomètres de Chartres. Il est ensuite l'élève de Gilbert de Poitiers, également membre de l'école de Chartres, qu'il cite plusieurs fois dans sa somme De ecclesiasticis officiis. Cette œuvre traite des rites ecclésiastiques, de leur fonction et de leur histoire. Dans le chapitre 120, Jean écrit au sujet des coutumes de plusieurs églises ${ }^{45}$ :

Il y a plusieurs églises où même les évêques et les archevêques jouent avec leurs clercs dans les cloîtres ou bien s'abaissent au jeu de la pelote. [...] Bien que ce jeu soit d'usage même dans de grandes églises, comme celle de Reims, il semble pourtant plus louable de ne pas jouer.

cf. J. Marenbon, From the Circle of Alcuin to the School of Auxerre, Cambridge, 1981 p. $116-138$

43. Son nom «Augustodunensis» est souvent attribué à la ville d'Autun, mais des références aux questions politiques de la région de l'Allemagne du Sud dans ses œuvres contredisent cette interptaion ( $f$. V. I.J. F. (1995, p. 95-128 (Hon Hius Augl and Religious Writers of the

44. Cf. C. J. Mews, \&Lits 44. Cf. C. J. Mews, «Liturgists and Dance in the Twelfth Century. The Witness of John Beleth and Sicard of Cremona», Church History, 78 (2009), p. 512-548.

45. «Sunt enim quedam ecclesie, ubi in claustris etiam ipsi episcopi vel archiepiscopi cum suis clericis ludunt, ut etiam descendant usque ad ludum pile. [...] Licet autem magne ecclesie ut Remensis hanc ludendi consuetudinem teneant, tamen non ludere laudabilius videtur» (Jean Beleth, Summa de ecclesiasticis officiis, éd. H. DouteIl, Turnhout, 1976, p. 223).
La description par Beleth du jeu que les évêques jouent avec leurs chanoines sera citée par notre auteur du XVIII ${ }^{\text {e }}$ siècle, Jean Lebeuf ${ }^{46}$. Ce dernier interprète ce passage comme la description d'un jeu profane, nommé aussi pelote, très populaire $\mathrm{au} \mathrm{Xv}^{\mathrm{e}}$ et $\mathrm{au} \mathrm{XVI} \mathrm{x}^{\mathrm{e}}$ siècle, parce que Beleth ne dit rien d'une danse. C'est un autre liturgiste, Sicard de Crémone, qui nous donne cette information supplémentaire selon laquelle le jeu de la pila - ou pelote - est une danse rituelle. Sicard est l'évêque de Crémone et a vécu en France durant ses études. Il précise dans son œuvre Mitralis de Officio (rédigée vers 1205$)^{47}$ :

C'est pourquoi, dans les cloîtres de quelques églises, les évêques pratiquaient avec leurs clercs la liberté de décembre et s'abaissaient même au jeu de la danse ou de la pelote, bien qu'il soit plus louable de ne pas jouer [...]. Et prends en considération que les gentils organisaient des danses pour leurs idoles, qu'ils adoraient par leurs voix et qu'ils servaient de tout leur corps [...], parce qu'ils comprenaient le mouvement des astres par des rondes, la connexion des éléments par l'enlacement des mains, les mouvements harmonieux des planètes par les mélodies chantées, les mouvements des planètes par les gestes des corps et le bruit de l'orage par le claquement des mains et le tapement des pieds. Mais ce qu'ils présentaient à leurs idoles, les adorateurs du seul Dieu l'ont transformé en sa louange.

La pelote est donc le mélange d'un jeu et d'une danse. Sicard fait converger les descriptions de Jean Beleth relatives aux pratiques des évêques et des chanoines, qui dansent en lançant une balle le jour de Noël ou de Pâques, avec les interprétations d'Honorius Augustodunensis sur l'inclusion de la théorie de l'harmonie des sphères dans un contexte chrétien. Il explique qu'autrefois les gentils dansaient pour honorer l'harmonie cosmique et que les chrétiens utilisent désormais les danses pour honorer Dieu, le maître de cette harmonie. Afin de légitimer les danses pour les chrétiens, Sicard cite des exemples bibliques déjà mentionnés par Honorius. Myriam avait dansé avec les femmes après la traversée de la mer rouge et David avait dansé devant l'Arche: la danse aurait donc une longue tradition judéo-chrétienne.

46. $C f$. J. Lebeuf, «Explication d'un terme...», p. $913 s q$.

47. «Inde est quod in claustris quarundam ecclesiarum etiam episcopi cum suis clericis decembrica libertate utuntur, descendentes etiam ad ludum choreae vel pilae, quamuis non ludere laudabilius sit [ ...]. Et attende, quod gentilitas as plausum idolorum choreas instituit, ut deos suos, et voce laudarent, et eis toto corpore servirent [...], nam per circuitionem intelligebant firmamenti revolutionem, per manuum complexionem elementorum connexionem, per melodias cantantium harmonias planetarum motiones, per corporum gesticulationes signorum vel planetarum motiones; per plausum manuum et strepitum pedum crepitationes tonitruorum. Sed quod illi suis idolis exhibuerunt, cultores unius Dei ad ipsius praeconia converterunt» (Sicard de Cremone, Mitralis, éd. G. Sarbak, L. Weinrich, Turnhout, 2008, p. 546) 
Une copie de la Summa de officiis ecclesiasticis de Guillaume d'Auxerre confirme l'interprétation de Sicard de Crémone en décrivant également des danses le soir de Pâques. Ce manuscrit, rédigé en Île-de-France au $\mathrm{XIII}^{\mathrm{e}}$ siècle, ne mentionne plus les exemples païens, mais fait directement allusion à la danse biblique de Myriam ${ }^{48}$.

Tous ces auteurs décrivent des danses, des jeux de ballon ou, comme à Auxerre, le mélange des deux pratiques. Le lancer d'un ballon dans la nef d'une cathédrale ne semblait pas bizarre uniquement à Lebeuf, mais aussi à d'autres chercheurs. Quel rapport pourrait-il y avoir entre la danse et le ballon?

\section{Le ballon comme symbole de l'harmonie de sphères}

Les commentaires des liturgistes, surtout celui de Sicard, montrent bien le lien entre le concept de l'harmonie des sphères, son acceptation par les auteurs chrétiens et la danse. Toutefois les chanoines à Auxerre ne se contentent pas de danser, ils lancent également un ballon, la pelote, que le nouveau chanoine doit offrir à l'église. Sicard ne donne pas d'explication sur le ballon. Grâce à la description du XvI ${ }^{e}$ siècle citée par Lebeuf et à un registre de la fin du XIV ${ }^{\mathrm{e}}$ siècle qui se trouve aux archives départementales d'Auxerre, nous avons deux informations supplémentaires sur ce ballon: il doit être assez grand de sorte qu'on doit le tenir à deux mains ${ }^{49}$, et le doyen le lance de sa main gauche ${ }^{50}$. La grandeur du ballon et le fait qu'il soit lancé de la main gauche ont provoqué de nombreuses spéculations.

Comme les chanoines dansent sur le labyrinthe de la cathédrale, ce dernier pourrait fournir une explication sur le lancer de la pelote. L'adaptation chrétienne du symbole du labyrinthe est déjà fréquente dans l'Antiquité tardive. Dès le haut Moyen Âge, en effet, une nouvelle forme de labyrinthe se développe dans les centres monastiques du Sud de l'Allemagne et du Nord de la France, notamment à Auxerre. Plusieurs manuscrits contiennent des représentations d'un labyrinthe circulaire à partir de la fin du IX ${ }^{\mathrm{e}}$ siècle $^{51}$. Les dessins de labyrinthe font souvent partie des computs qui aident à calculer la date exacte de Pâques. Ils deviennent l'exemple des grands labyrinthes, qui sont gravés sur le sol des nefs de certaines cathédrales en France aux XII et XIII ${ }^{\mathrm{e}}$ siècles.

48. Guillaume d’AuXerRe, Summa de officiis ecclesiasticis, éd. F. Fischer, Dissertation, Cologne, 2007, p. 81.

49. «Fuit ordinatum quod Pilota fiat minor solito, tamen quod non possit comprehendi seu apprehendi una sola manu hominis » (Bibliothèque municipale d'Auxerre, G 1798 , fol. 285).

50. «Manu prehensis» (J. LeBeuf, «Explication d'un terme...», p. 921)

51. Cf. C. WRIGHT, The Maze . p. 20-27.

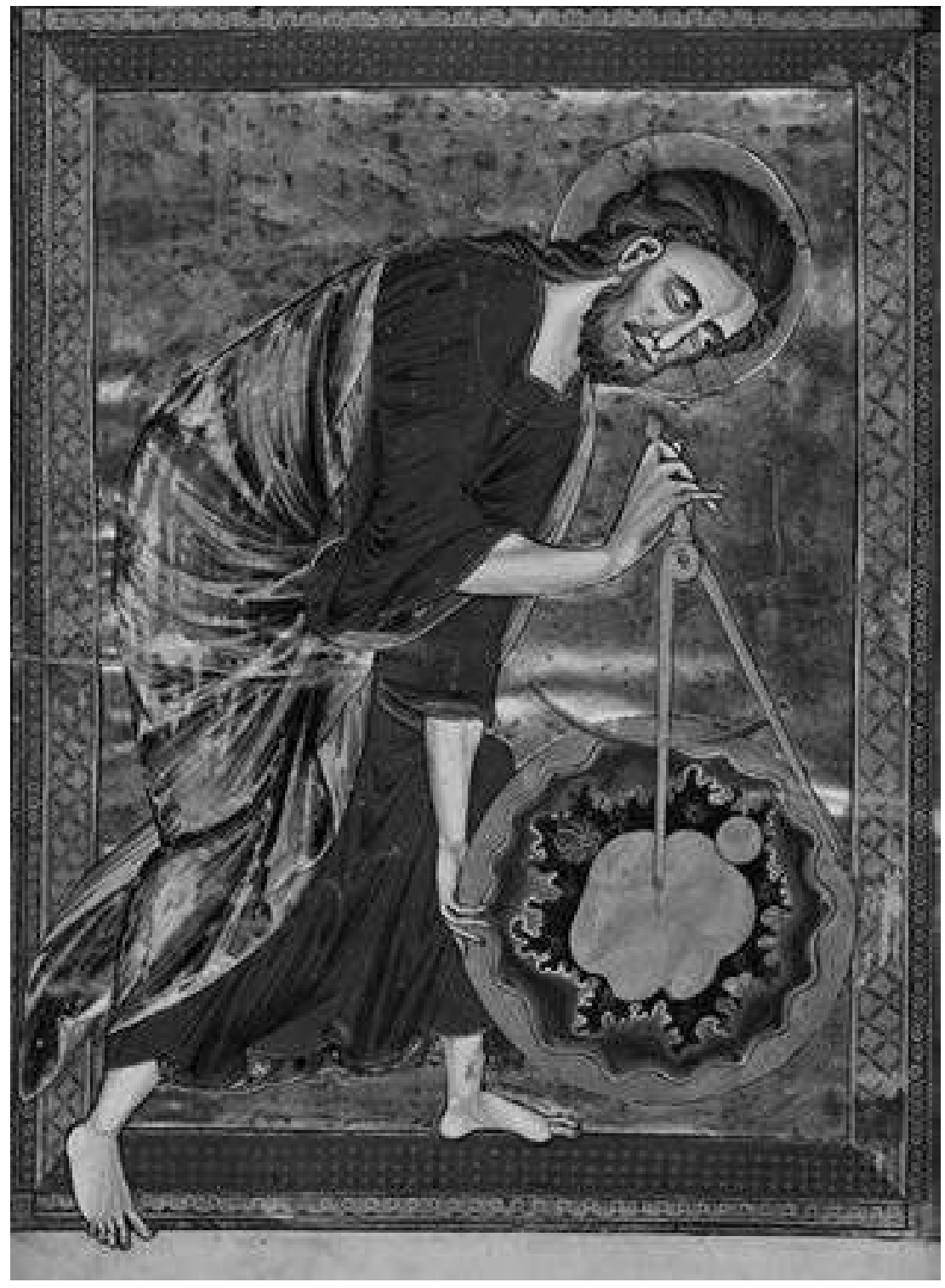

Fig. 1. Bible moralisée, Vienne, Österreichische Nationalbibliothek, Vindobonensis 2554 (France, $\mathrm{XIII}^{\mathrm{e}}$ siècle) 


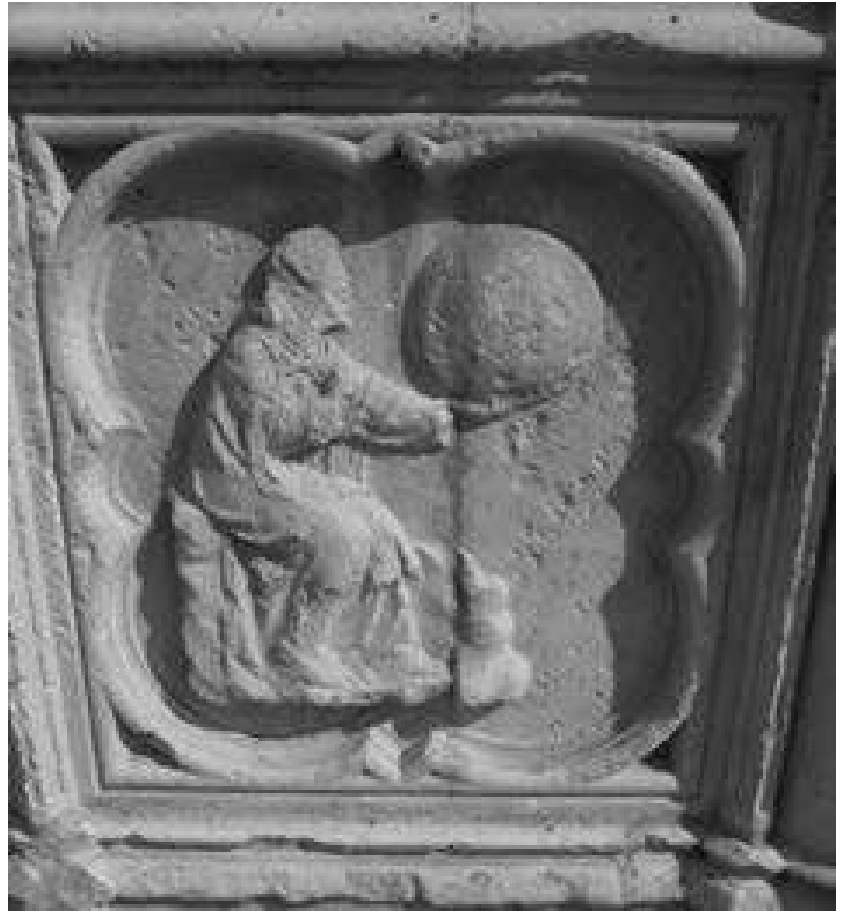

Fig. 2. Cathédrale Saint-Étienne d'Auxerre, portail ouest (XIII siècle) (ㅇ P. Knäble).

Au labyrinthe est associé le mythe grec de Thésée, qui tue le Minotaure dans le labyrinthe de $\operatorname{Cnossos}^{52}$. Avant qu'il n'y pénètre, Thésée reçoit d'Ariane deux balles: une pelote de fil, le fameux fil d'Ariane, afin de s'échapper du labyrinthe, et une balle de glaise avec lequel il vainc le Minotaure en le lançant dans sa bouche ${ }^{53}$. Avec l'adaptation du mythe dans un contexte chrétien, la victoire de Thésée sur le minotaure est interprétée comme la victoire du Christ sur Satan et sa Résurrection le jour de Pâques. La pelote de fil signifierait la nature humaine et la balle de glaise la nature divine du Christ ${ }^{54}$.

D'autres auteurs ont proposé une interprétation du ballon comme symbole du soleil levant qui, depuis le début du christianisme, est également

52. Sur la combinaison du labyrinthe et de mythe de Thésée, $c f$. W. Haubrichs, «Error inextricabilis. Form und Funktion der Labyrinthabbildung in mittelalterlichen Handschriften », dans C. MEIER, U. RuBERG éd., Text und Bild.Aspekte des Zusammenwirkens zweier Künste im Mittelalter und früher Neuzeit, Wiesbaden, 1980, p. 134-137.

53. $C f$. M. Eisenberg, Performing..., sans pagination.

54. Cf. Р. Фоов, The Idea of the Labyrinth from Classical Antiquity through the Middle Ages, New York, 1990, p. 125; G. RoHmann, Tanzwut...,p. 244. une image du Christ. Ce dernier, qui revient de l'enfer le jour de Pâques, est symbolisé par le soleil de la résurrection (sol resurrectionis ou sol salutis Christi) ${ }^{55}$. Craig Wright émet l'hypothèse que le lancer de la pelote pourrait avoir plusieurs significations pour le clergé: «Thus the flying pilota symbolized several things: the instrument of salvation derived from the Greek myth, the rising sun from the folkloric legend, and the harmony of the spheres from classical philosophy ${ }^{56}$.»

Mais pourquoi le ballon doit-il être si grand et pourquoi doit-il être lancé de la main gauche? Pour compléter ces spéculations, je vais tenter de démontrer que le ballon pourrait aussi faire directement allusion à l'harmonie des sphères ${ }^{57}$. Craig Wright reproduit dans son livre une image d'une bible moralisée du XIII ${ }^{\mathrm{e}}$ siècle, qui montre la forme des labyrinthes en cercles dans les manuscrits monastiques (fig. 1) ${ }^{58}$. L'enluminure met en scène Dieu comme architecte de l'univers. Il tient un compas de sa main droite, avec lequel il crée de manière mathématique un cercle, signe de l'harmonie et de la perfection divine ${ }^{59}$. Le soleil et d'autres planètes font comprendre que l'image représente la création du monde ${ }^{60}$. Dieu tient ou roule le monde de sa main gauche. Si l'on considère la grandeur de la boule, qui va des pieds au genou, la lancer d'une seule main semble, sinon impossible, du moins très difficile.

Une autre représentation de la création du monde, qui montre Dieu tenant le globe terrestre de la main gauche, se trouve aussi sur la façade occidentale de la cathédrale d'Auxerre (fig. 2). Un bas-relief du XIII ${ }^{\mathrm{e}}$ siècle montre Dieu assis, qui regarde son œuvre terminée, le globe terrestre. Ce globe a également une taille relativement grande. D'après René Fourrey, on a l'impression «qu'il se dispose à lancer [le globe] à travers l'espace ${ }^{61}$ », un geste qui devait inspirer les chanoines de la cathédrale pour le lancer de la pelote le jour de Pâques. Le lien avec la création de l'univers, qui est par sa forme sphérique le signe d'une harmonie parfaite, est déjà exprimé par la danse et l'hymne Victimae paschali laudes, et souligné par le lancer

55. Cf. C. Wright, The Maze..., p. 142 ; M. EISENBERG, Performing..., sans pagination.

56. C. WRIGHT, The Maze..., p. 142

57. Je suis ici une idée de R. Fourrey, Dans la cathédrale Saint-Étienne d'Auxerre, Auxerre, 1934, p. 155.

58. C. Wright, The Maze..., p. 22 (enluminure d'une Bible moralisée, France, XIII ${ }^{e}$ s., Vienne, Österreichische Nationalbibliothek, Codex 2554, fol. 4).

59. Cf. C. Wright, The Maze..., p. 23; F. BoesPflug, «Le créateur au compas. Deus geometra dans l'art d'Occident», Mircologus, 19 (2011), p. 113-130.

60. Pour d'autres représentations du Dieu au compas, $c f$. F. BoEsPflug, «Le créateur...», p. 117-127. Une fresque à l'abbaye Saint-Savin du XI ${ }^{\circ} \mathrm{s}$. montre une scène de la création du monde dans laquelle Dieu tient deux ballons de ses mains. De sa main droite il lance (ou attrape) le soleil, dans sa main gauche se trouve la boule représentant la lune: cf. B. MerdRIGnaC, Le Sport au Moyen Âge, Rennes, 2002, p. 197.

61. R. FourReY, Dans la cathédrale..., p. 155 
du ballon le jour de Pâques. Jusqu'à la fin du Xvi ${ }^{\mathrm{e}}$ siècle, le jour de Pâques est le début officiel de l'année et symbolise, dans un contexte plus général, d'une part la création du monde, d'autre part la résurrection du Christ, c'està-dire la victoire du Christ sur le diable et son retour de l'enfer qui permet la restauration de l'harmonie céleste.

L'interprétation de Jean Lebeuf et d'autres auteurs savants du $\mathrm{XVIII}^{\mathrm{e}}$ siècle, qui qualifient les danses dans les églises de pratiques bizarres ou de cérémonies extravagantes d'une époque gothique, manifeste leur manque de compréhension de ce phénomène. La danse est un sujet ambivalent dans le discours des théologiens et un moyen d'exprimer l'harmonie (l'imitation de la danse des anges) ou la disharmonie (les danses diaboliques). L'association d'une danse ecclésiastique avec le lancer d'un ballon dans la cathédrale d'Auxerre le jour de Pâques est la mise en scène de l'idée de l'harmonie des sphères transposée à un contexte chrétien et discutée dans les écoles cathédrales du Moyen Âge. La pelote d'Auxerre n'est pas une «grande bizarrerie» mais un rituel très élaboré, par lequel les chanoines de la cathédrale expriment l'harmonie restituée par la victoire du Christ sur le diable.

Philip KNÄBLE - Bielefeld Graduate School in History and Sociology (BGHS), Universität Bielefeld

\section{L'harmonie des sphères et la danse dans le contexte clérical au Moyen Âge}

L'Église, et surtout l'Église médiévale, est considérée comme une institution complètement opposée à la danse. Pourtant il y avait des églises cathédrales, par exemple celle d'Auxerre, où les chanoines eux-mêmes dansaient dans l'église pendant les fêtes religieuses. L'article discute ces pratiques de danse en relation avec la réception de la philosophie néoplatonicienne et son concept de l'harmonie des sphères dans les écoles cathédrales. En considérant la danse comme une mise en scène de l'harmonie des sphères, les chanoines intégrèrent celle-ci dans leurs pratiques de dévotion.

Auxerre - danse - harmonie des sphères - liturgistes - pelote

\section{Dance and The Harmony of the Spheres in the Medieval Clerical Context}

The Church, especially the medieval Church, is known as an institution completely opposed to dancing. Nevertheless there existed some cathedral churches, for example at Auxerre, where even the canons danced in the church during religious feasts. This article discusses the ecclesiastical dances in relation to the reception of Neo-Platonist philosophy and its concept of the harmony of the spheres at the cathedral schools. Viewing the dance as a performative expression of the harmony of the spheres the canons incorporate the dance as a practice of devotion.

Auxerre - dance - harmony of the spheres - liturgists - pelota 